\title{
Eigenvalue Variation in MIMO OFDM Systems
}

\author{
Krishna P. Kongara Peter J. Smith Lee M. Garth \\ Department of Electrical and Computer Engineering, \\ University of Canterbury, Christchurch, New Zealand
}

\begin{abstract}
In this paper we consider the variation of eigenvalues and eigenvalue sums across the frequency bins of a MIMO OFDM system. In particular, we consider the changes in the ordered eigenvalues and the eigenvalue sum or link gain between two distinct frequency bins. The size of such changes has an important effect both on system performance and design. In addition these results have applications in other areas, including temporal variation, feedback delay and channel estimation. Novel results presented include distributions and moments for changes in link gain and the maximum eigenvalue and autocorrelation functions for the link gain and maximum eigenvalue. Furthermore, some very simple approximate results for the ordered eigenvalue differences are presented and the accuracy of the analysis is verified by Monte Carlo simulations.
\end{abstract}

Keywords: MIMO OFDM, eigenvalues, frequency autocorrelation function, link gain.

\section{INTRODUCTION}

In this paper we consider a multiple-input multiple-output orthogonal frequency division multiplexing (MIMO OFDM) system operating over frequency selective channels. In particular, we focus on the eigenvalues of the complex Wishart matrices defined for each OFDM subcarrier and investigate their variation across frequency. Since the throughput and performance of such a system are heavily dependent on the eigenvalues, the results and analysis presented have many applications. Examples include the performance of beamforming systems which is governed by the maximum eigenvalue and the link gain which is characterized by the sum of the eigenvalues.

Specifically, we consider the differences between eigenvalues and between eigenvalue sums across the OFDM frequency bins in both Rayleigh and Rician fading channels. This leads us to consider eigenvalue autocorrelation functions (ACFs) and various joint distributions in frequency. The main contributions of the paper are the following:

- Maximum eigenvalue results including the joint probability density function (PDF) of the maximum eigenvalue in two frequency bins, the ACF of the maximum eigenvalue, the distribution and variance of the difference between the two maximum eigenvalues.

- Link gain results including the PDF, the cumulative distribution function (CDF) and variance of the link gain difference, and the ACF of the link gain.

- Simple approximations to the ACF of the maximum eigenvalue and to the variance of the maximum eigenvalue difference.
Although the focus of this work is on the variation over frequency, exactly the same approach can be used to characterize changes in eigenvalues over time or space. For example, the frequency ACFs developed can be converted to temporal ACFs by replacing the channel ACF over frequency with the channel ACF in time. This leads to work on the time varying nature of the eigenvalues [1], [2]. Such temporal variation is important in the analysis of adaptive MIMO systems with feedback delay [3], [4]. Another example is channel estimation where the effects of imperfect channel state information can be modeled by channel estimates which are correlated with the true channel values. Again, the correlation between the true and estimated channels can be used to replace the frequency correlation and lead to useful results on channel estimation [5], [3]. Finally, many limited feedback OFDM systems have been proposed to decide a single transmission strategy for a contiguous block of frequency bins. A simple way to decide the number of carriers to include in a block would be to compare the width of the block with the standard deviation of the eigenvalue differences. This gives a good indication of how widely the eigenvalues will vary across the block for wireless fading channels [6], [7].

This paper is organised as follows. In Sec. II we define the MIMO OFDM system model. In Sec. III we introduce the particular eigenvalue metrics we study in this paper. In Sec. IV we analytically derive the distributions and moments for certain of these metrics for MIMO OFDM systems in Rayleigh and Rician fading channels. In Sec. V we discuss our simulation results and draw our conclusions in Sec. VI.

\section{MIMO OFDM SYSTEM MODEL}

Consider a standard MIMO OFDM system where $\boldsymbol{H}_{i}$ represents the $n_{R} \times n_{T}$ channel matrix for the $i$-th subcarrier, $n_{R}$ is the number of receive antennas, $n_{T}$ is the number of transmit antennas and there are $N$ subcarriers. We assume zero spatial correlation, so the entries of $\boldsymbol{H}_{i}$ are independent. Furthermore, we assume independent and identically distributed (i.i.d.) Rayleigh or Rician fading with normalized power so the entries of $\boldsymbol{H}_{i}$, denoted $h_{r s}^{(i)}$, are i.i.d. Gaussians satisfying $\mathrm{E}\left[\left|h_{r s}^{(i)}\right|^{2}\right]=1$. For the Rician case, the channel has the specific form $\boldsymbol{H}_{i}=\sqrt{\frac{K}{1+K}} \boldsymbol{H}_{i, \mathrm{sp}}+\sqrt{\frac{1}{1+K}} \boldsymbol{H}_{i, \mathrm{sc}}$ where $K$ is the Rician K-factor and $\boldsymbol{H}_{i \text {,sp }}$ and $\boldsymbol{H}_{i \text {,sc }}$ are unit power specular and scattered matrices, respectively [8].

Across the frequency bins we denote the auto-correlation function (ACF) of the channel coefficients by

$$
\rho_{h}(k)=\mathrm{E}\left[h_{r s}^{(1)} h_{r s}^{*(k+1)}\right]-\mathrm{E}\left[h_{r s}^{(1)}\right] \mathrm{E}\left[h_{r s}^{*(k+1)}\right]
$$


TABLE I

DELAy SPECTRA AND FREQUENCY AUtOCORRELATION FunCTIONS.

\begin{tabular}{|c|c|c|}
\hline $\begin{array}{c}\text { Delay } \\
\text { Spectrum }\end{array}$ & $\begin{array}{c}\text { Delay Spectrum, } \\
P_{h}(\tau)\end{array}$ & $\begin{array}{c}\text { Frequency ACF, } \\
\rho_{h}(k)\end{array}$ \\
\hline Exponential & $\frac{1}{\tau_{0}} e^{\left[-\tau / \tau_{0}\right]}$ & $\frac{1}{1+j 2 \pi k \Delta f \tau_{0}}$ \\
\hline Gaussian & $\frac{1}{\sqrt{2 \pi} \tau_{0}} e^{\left[-\frac{1}{2}\left(\tau / \tau_{0}\right)^{2}\right]}$ & $e^{\left[-2\left(\pi k \Delta f \tau_{0}\right)^{2}\right]}$ \\
\hline Double-Spike & $\frac{1}{2}\left[\delta(\tau)+\delta\left(\tau-2 \tau_{0}\right)\right]$ & $\frac{1}{2}\left[1+e^{\left(-4 j \pi k \Delta f \tau_{0}\right)}\right]$ \\
\hline Uniform & $\begin{array}{ll}\frac{1}{\tau_{0}}, & \text { if }|\tau| \leq \frac{\tau_{0}}{2} \\
0, & \text { otherwise }\end{array}$ & $\operatorname{sinc}\left(k \Delta f \tau_{0}\right)$ \\
\hline
\end{tabular}

where $h_{r s}^{(b)}$ indicates the $r s$-th channel coefficient in bin $b$. If the frequency spacing between adjacent bins is denoted by $\Delta f$, the two carriers in (1) are separated by $k \Delta f$. The delay spectra considered in this paper include the classic Jakes model [9], a Gaussian power delay profile (PDP), a double-spike power delay profile and a uniform power delay profile [10]. These models give the ACF results specified in Table I [10]. The parameter, $\tau_{0}$, is the root mean square delay spread.

The performance of a MIMO OFDM system is largely governed by the eigenvalues of the channel correlation matrices, $\boldsymbol{H}_{i} \boldsymbol{H}_{i}^{\dagger}$, where $\dagger$ represents the conjugate transpose. We denote these eigenvalues by $\lambda_{1}^{(i)}>\lambda_{2}^{(i)}>\cdots>\lambda_{m}^{(i)}$ where $m=\min \left(n_{R}, n_{T}\right)$ and $n=\max \left(n_{R}, n_{T}\right)$.

\section{Eigenvalue Metrics}

In this paper we are concerned with the variation in eigenvalues across the frequency domain. In particular we consider two frequency bins in the OFDM system and investigate the difference $Z=X-Y$, which measures the change in some eigenvalue metric across frequency. Specifically, we look at the following cases

$$
\begin{aligned}
Z_{\max } & =\lambda_{1}^{(1)}-\lambda_{1}^{(k+1)} \\
Z_{\min } & =\lambda_{m}^{(1)}-\lambda_{m}^{(k+1)} \\
Z_{j} & =\lambda_{j}^{(1)}-\lambda_{j}^{(k+1)} \\
Z_{\text {sum }} & =\sum_{j=1}^{m} \lambda_{j}^{(1)}-\sum_{j=1}^{m} \lambda_{j}^{(k+1)} .
\end{aligned}
$$

These cases give, respectively, the differences between the maximum eigenvalues, minimum eigenvalues, $j$-th eigenvalues and the total link gains. For ease of notation, the dependence of the $Z$ metrics on $k$ is not shown.

In all cases we have $\mathrm{E}[Z]=0$, since the channel process is stationary across frequency. Hence, the key summary measure of interest is the variance, $\mathrm{E}\left[Z^{2}\right]$. Note that $\operatorname{var}(X-Y)=$ $\operatorname{var}(X)+\operatorname{var}(Y)-2 \operatorname{cov}[X, Y]$, so that the correlation between $X$ and $Y$ is given by

$$
\operatorname{corr}(X, Y)=\frac{\operatorname{var}(X)+\operatorname{var}(Y)-\operatorname{var}(X-Y)}{2 \sqrt{\operatorname{var}(X) \operatorname{var}(Y)}} .
$$

In all cases of interest, $X$ and $Y$ are identically distributed and $\operatorname{corr}(X, Y)$ is the ACF of each eigenvalue metric over frequency, denoted by $\rho_{k}$. Hence, we have

$$
\rho_{k}=1-\frac{\operatorname{var}(X-Y)}{2 \operatorname{var}(X)} .
$$

Now the marginal statistics, such as $\mathrm{E}[X]$ and $\operatorname{var}(X)$, can be computed using standard results [11], [12], [13], [14]. Thus, we can find the ACF from $\operatorname{var}(X-Y)$ and vice-versa.

In addition to the variance, $\operatorname{var}(Z)$, and $\mathrm{ACF}, \rho_{k}$, we also consider the PDF and CDF of $Z$ and the joint distributions of the eigenvalues in the two bins.

\section{ANALYSIS}

Here, we focus on $Z_{\text {sum }}$ and $Z_{\max }$ since analytical progress is possible for these random variables. Note that the techniques used for the maximum eigenvalue difference, $Z_{\max }$, can also be applied to the minimum eigenvalue case. Such extensions are straightforward and are mentioned only briefly.

\section{A. $Z_{\text {sum }}$ : Rician Fading}

In this section, we consider the variable

$$
\begin{aligned}
Z_{\text {sum }} & =\sum_{j=1}^{m}\left[\lambda_{j}^{(1)}-\lambda_{j}^{(k+1)}\right] \\
& =\sum_{i, j}\left[\left|\left(\boldsymbol{H}_{1}\right)_{i, j}\right|^{2}-\left|\left(\boldsymbol{H}_{k+1}\right)_{i, j}\right|^{2}\right]
\end{aligned}
$$

where $(\boldsymbol{H})_{i, j}$ denotes the $(i, j)$-th entry of matrix $\boldsymbol{H}$. Note that $Z_{\text {sum }} / m$ is the average eigenvalue difference and $Z_{\text {sum }}$ is the difference between the link gains.

For convenience we consider the variable $q=(1+K) Z_{\text {sum }}$. From (5) we see that $q$ is a quadratic form in complex Gaussian random variables. Such a quadratic form has been studied in [15] including its PDF, CDF, mean and variance. Invoking the results in [15], we can derive the following PDF for $q \in$ $(-\infty, \infty)$

$$
\begin{aligned}
& f_{q}(x)=2^{-m n} e^{-3 \beta} \sum_{k=0}^{\infty} \frac{(2 \beta)^{k}}{k !} \\
& \times \sum_{r=0}^{k+m n-1} \frac{\alpha^{k+m n-r} x^{k+m n-r-1} e^{-\alpha|x|} L_{r}^{(m n-1)}(-\beta)}{(k+m n-r-1) ! 2^{r}}
\end{aligned}
$$

where $\alpha=\left(1-\left|\rho_{h}(k)\right|^{2}\right)^{-1 / 2}$ and $\beta=m n K /\left[2\left(1+\rho_{h}(k)\right)\right]$. The function $L_{r}^{\alpha}(-b)$ is the generalized Laguerre polynomial [16] given by $L_{r}^{\alpha}(-b)=\sum_{k=0}^{r}\left(\begin{array}{l}r+\alpha \\ r-k\end{array}\right) b^{k} / k !$. The corresponding $\mathrm{CDF}$ is

$$
F_{q}(x)= \begin{cases}R(x), & \text { if } x<0 \\ 1-R(x), & \text { if } x \geq 0\end{cases}
$$

where the function $R(\cdot)$ is defined by

$$
\begin{aligned}
R(x) & =2^{-m n} e^{-3 \beta} \sum_{k=0}^{\infty} \frac{(2 \beta)^{k}}{k !} \\
& \times \sum_{r=0}^{k+m n-1} \frac{\Gamma(k+m n-r, \alpha|x|) L_{r}^{(m n-1)}(-\beta)}{(k+m n-r-1) ! 2^{r}}
\end{aligned}
$$


with $\Gamma(a, x)=\int_{x}^{\infty} t^{a-1} e^{-t} d t$. The moments of $q$ are simpler with $\mathrm{E}[q]=0$ by symmetry and variance $\sigma_{q}^{2}$ given by

$$
\sigma_{q}^{2}=2 m n\left\{2 K[1-\operatorname{Re}(\rho)]+1-|\rho|^{2}\right\} .
$$

\section{B. $Z_{\text {sum }}$ : Rayleigh Fading}

In the Rayleigh fading case, $K=0$ and (6) simplifies considerably since $\beta=0$. The resulting PDF is

$f_{q}(x)=2^{-m n} \sum_{r=0}^{m n-1} \frac{\alpha^{m n-r} x^{m n-r-1} e^{-\alpha|x|}}{(m n-r-1) ! 2^{r}}\left(\begin{array}{c}r+m n-1 \\ r\end{array}\right)$.

The CDF is also of the form given in (7) with

$$
R(x)=2^{-m n} \sum_{r=0}^{m n-1} \frac{\Gamma(m n-r, \alpha|x|)}{(m n-r-1) ! 2^{r}}\left(\begin{array}{c}
r+m n-1 \\
r
\end{array}\right) .
$$

The tail probabilities have an even simpler form. Using the series expansion for $\Gamma(m n-r, \alpha|x|)$ for large values of $\alpha|x|$, we have the result [16]

$$
\begin{aligned}
R(x)= & \sum_{r=0}^{m n-1}\left(\begin{array}{c}
r+m n-1 \\
r
\end{array}\right) \frac{(\alpha|x|)^{m n-r-1} e^{-\alpha|x|}}{2^{m n+r}(m n-r-1) !} \\
& \times\left[1+\frac{m n-r-1}{\alpha|x|}+\cdots\right] \\
= & \frac{(\alpha|x|)^{m n-1} e^{-\alpha|x|}}{2^{m n}(m n-1) !}\left[1+\frac{m n-1}{\alpha|x|}\left(1+\frac{m n}{2}\right)\right. \\
& \left.+o\left(\frac{1}{\alpha|x|}\right)\right] .
\end{aligned}
$$

For values of $\alpha|x|$ large enough that terms in $(\alpha|x|)^{-1}$ are negligible, the tail probabilities are well approximated by the simple result

$$
R(x) \approx \frac{(\alpha|x|)^{m n-1} e^{-(\alpha|x|)}}{2^{m n}(m n-1) !} .
$$

From (9), the variance is

$$
\sigma_{q}^{2}=2 m n\left(1-|\rho|^{2}\right) .
$$

Note that relative changes are of the form $Z_{\mathrm{rel}}=(X-$ $Y) / X$. To obtain the distribution of $Z_{\text {rel }}$, note that $P\left(Z_{\text {rel }} \leq\right.$ $z)=P([1-z] X-Y \leq 0)$. Hence, the distribution of $Z_{\mathrm{rel}}$ is given by a very similar calculation to that required for $Z=$ $X-Y$ as outlined in [15].

\section{C. $Z_{\max }$ : Rayleigh Fading}

The joint density of $\left(\lambda_{1}^{(1)}, \ldots, \lambda_{m}^{(1)}, \lambda_{1}^{(k+1)}, \ldots, \lambda_{m}^{(k+1)}\right)$ is derived in [11]. This provides a general framework for the exact calculation of the moments, PDFs and CDFs associated with the $Z$ metrics in (2). In practice, however, this often leads to multiple numerical integrals and may not be a practical approach. Hence, special cases and approximate solutions are worth pursuing. Special cases of interest include the maximum eigenvalue and the minimum eigenvalue.

In [17] the joint distribution function of $\left(\lambda_{1}^{(1)}, \lambda_{1}^{(k+1)}\right)$ was derived as

$$
F_{k}(x, y)=C\left|A_{i j}(x, y)\right|
$$

where $C=\left(1-\left|\rho_{h}(k)\right|^{2}\right)^{-m}\left|\rho_{h}(k)\right|^{-m(n-1)} /\left[\Pi_{k=1}^{m}(n-\right.$ $k) !(m-k) !]$ and $\left|A_{i j}(x, y)\right|$ represents the determinant of the $m \times m$ matrix $\boldsymbol{A}(x, y)$ with $(i, j)$-th element

$$
\begin{aligned}
A_{i j}(x, y) & =\sum_{k=0}^{\infty} \frac{\mu^{k+\frac{n-m}{2}}}{\delta^{n-m+j+k} \delta^{n-m+i+k} k !(k+n-m) !} \\
& \times \gamma(n-m+j+k, \delta y) \gamma(n-m+i+k, \delta x) .
\end{aligned}
$$

In (16), $\mu=\left|\rho_{h}(k)\right|^{2}\left(1-\left|\rho_{h}(k)\right|^{2}\right)^{-2}, \delta=\left(1-\left|\rho_{h}(k)\right|^{2}\right)^{-1}$ and incomplete gamma function $\gamma(\alpha, \beta)=\int_{0}^{\beta} t^{\alpha-1} e^{-t} d t$. To obtain the ACF of the maximum eigenvalue or find $\operatorname{var}\left(Z_{\max }\right)$, it suffices to compute

$$
\mathrm{E}\left[\lambda_{1}^{(1)} \lambda_{1}^{(k+1)}\right]=\int_{0}^{\infty} \int_{0}^{\infty} x y f_{k}(x, y) d x d y
$$

where $f_{k}(x, y)=\frac{\partial^{2}}{\partial x \partial y} F_{k}(x, y)$ is the joint PDF of $\left(\lambda_{1}^{(1)}, \lambda_{1}^{(k+1)}\right)$. It is straightforward to show that

$$
\begin{gathered}
\mathrm{E}\left[\lambda_{1}^{(1)} \lambda_{1}^{(k+1)}\right]=\int_{0}^{\infty} \int_{0}^{\infty} P\left(\lambda_{1}^{(1)}>x, \lambda_{1}^{(k+1)}>y\right) d x d y \\
=\int_{0}^{\infty} \int_{0}^{\infty}\left[F_{k}(x, y)-F^{(1)}(x)-F^{(1)}(y)-1\right] d x d y
\end{gathered}
$$

where $F^{(1)}(x)=P\left(\lambda_{1}^{(1)}<x\right)$ is given in [12], [14]. Hence, using (15) and results in [12], [14], we have the ACF of the maximum eigenvalue via a double numerical integral.

A very similar approach can be used for the minimum eigenvalue. Distributional results for these two cases can also be obtained with similar complexity. For example, the probability

$$
P\left(Z_{\max }<z\right)=\int_{-\infty}^{\infty} \int_{x-z}^{\infty} f_{k}(x, y) d y d x
$$

requires a double numerical integral over the joint density.

Note that $f_{k}(x, y)$ is not reported in [17] but is simple to obtain as follows. Differentiating (15) with respect to $x$ gives

$$
\frac{\partial}{\partial x} F_{k}(x, y)=C \sum_{s=1}^{m}\left|A_{i j}^{(r)}(x, y)\right|
$$

where $\left(A_{i j}^{(r)}(x, y)\right)$ is the matrix $\left(A_{i j}(x, y)\right)$ after differentiation of row $r$ with respect to $x$. Similarly, differentiating (20) with respect to $y$ gives

$$
f_{k}(x, y)=C \sum_{r=1}^{m} \sum_{s=1}^{m}\left|A_{i j}^{(r, s)}(x, y)\right|
$$

where the $s$-th column has been differentiated with respect to $y$. The elements, $A_{i j}^{(r, s)}(x, y)$, are defined by

$$
A_{i j}^{(r, s)}(x, y)=\sum_{k=0}^{\infty} c_{k, i, j} w_{1}(k, j, y) w_{2}(k, i, x)
$$




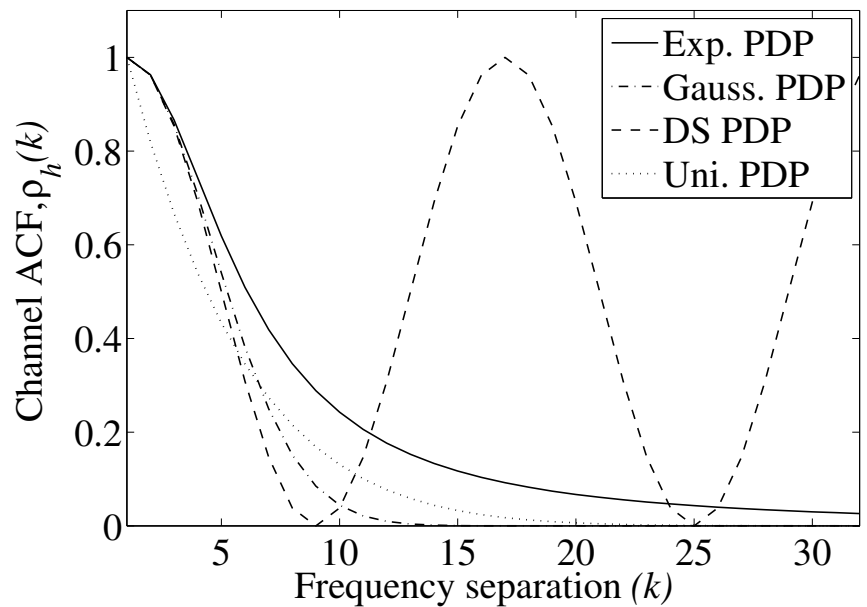

Fig. 1. Frequency autocorrelation function of the channel with $\tau_{0} \Delta f=$ 0.01325 .

where $c_{k, i, j}=\mu^{k+\frac{n-m}{2}} \delta^{-n+m-j-k} \delta^{-n+m-i-k} /[k !(k+n-$ $m) !$ and

$$
\begin{aligned}
& w_{1}(k, j, y)= \begin{cases}\gamma(n-m+j+k, \delta y), & j \neq s \\
\delta^{n-m+j+k} y^{n-m+j+k-1} e^{-\delta y}, & j=s\end{cases} \\
& w_{2}(k, i, x)=\left\{\begin{array}{ll}
\gamma(n-m+i+k, \delta x), & i \neq r \\
\delta^{n-m+i+k} x^{n-m+i+k-1} e^{-\delta x}, & i=r
\end{array} .\right.
\end{aligned}
$$

\section{Simulation Results}

In Fig. 1 we show the four types of channel ACF over frequency corresponding to the exponential (Exp.), Gaussian (Gauss.), double-spike (DS) and uniform (Uni.) power delay profiles [10]. In all cases the product of $\Delta f$ and the rms delay spread $\tau_{o}$ is set to 0.03125 which matches the HyperLan 2 standard. The DS ACF is fundamentally different to the decaying nature of the other models and is used to model hilly terrain [18]. Of the other ACFs, the exponential power delay profile results in the slowest decay rate over frequency.

For these four models the variance of $Z_{\max }$ is plotted against frequency spacing in Fig. 2 for a $(4,4)$ system. Rayleigh fading is assumed for Figs. 2-5. As expected, the DS scenario oscillates, and the exponential case is the slowest to rise of the remaining three models. The variance curves level off around 30 which is $2 \operatorname{var}\left(\lambda_{\max }^{(1)}\right)$. Also shown in Fig. 2 is an approximate variance result, discussed in more detail below.

Figure 3 shows the effect of system size on $\operatorname{var}\left(Z_{\max }\right)$ for an exponential power delay profile. There are no surprises here with the larger systems being more variable, and the trends are all very similar. Figure 4 shows the ACFs of the four ordered eigenvalues in a $(4,4)$ system for the exponential power delay profile. The dominant eigenvalues take longer to decorrelate, but in all cases, $\rho_{k} \leq 0.5$ after a separation of seven or more bins. Also plotted in Fig. 4 is the squared magnitude of the channel ACF, $\left|\rho_{h}(k)\right|^{2}$. It is interesting that this simple expression gives a tight bound on the ACF of the maximum eigenvalue and progressively weaker bounds on the ACFs of the lesser eigenvalues.

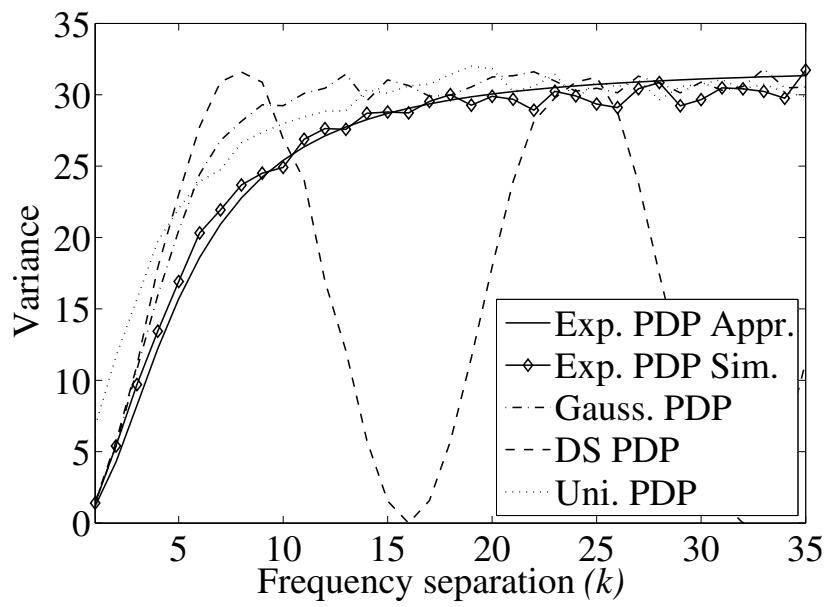

Fig. 2. Variance of $Z_{\max }$ vs. frequency separation in a $(4,4)$ MIMO OFDM system with $\tau_{0} \Delta f=0.01325$.

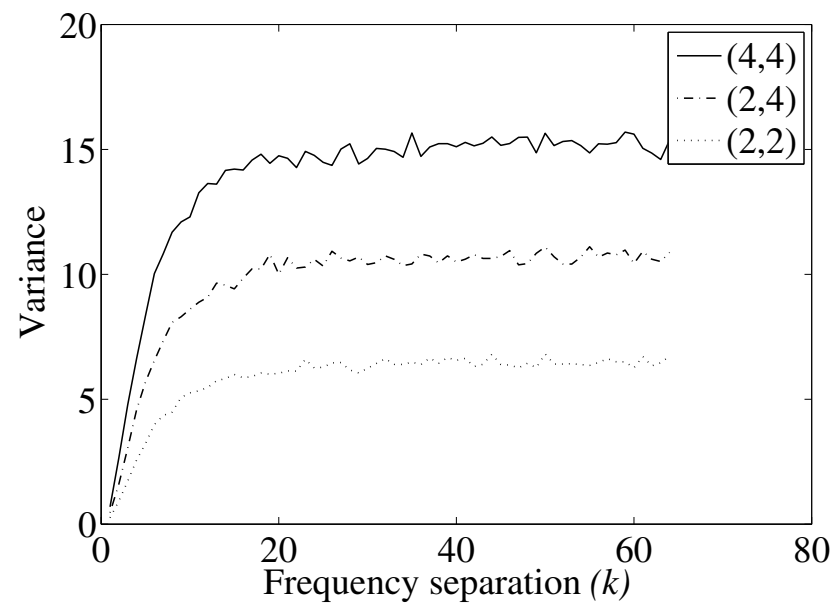

Fig. 3. Variance of $Z_{\max }$ vs. frequency separation in a MIMO OFDM system with exponential PDP, $\tau_{0} \Delta f=0.01325$ and varying system sizes.

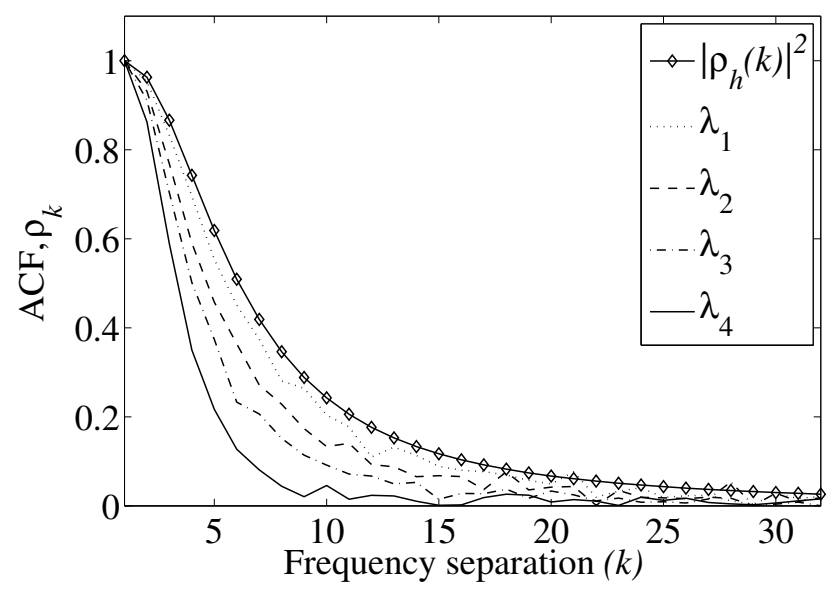

Fig. 4. Frequency autocorrelation function for the eigenvalues in a $(4,4)$ MIMO OFDM system system with exponential PDP and $\tau_{0} \Delta f=0.01325$. 


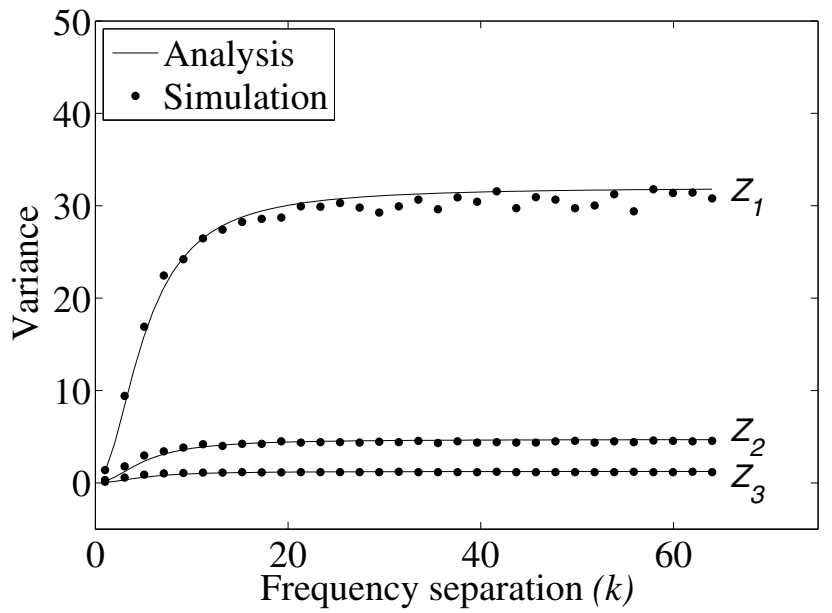

Fig. 5. Variances of $Z_{1}, Z_{2}$ and $Z_{3}$ vs. frequency separation in a $(4,4)$ MIMO OFDM system with exponential PDP and $\tau_{0} \Delta f=0.01325$.

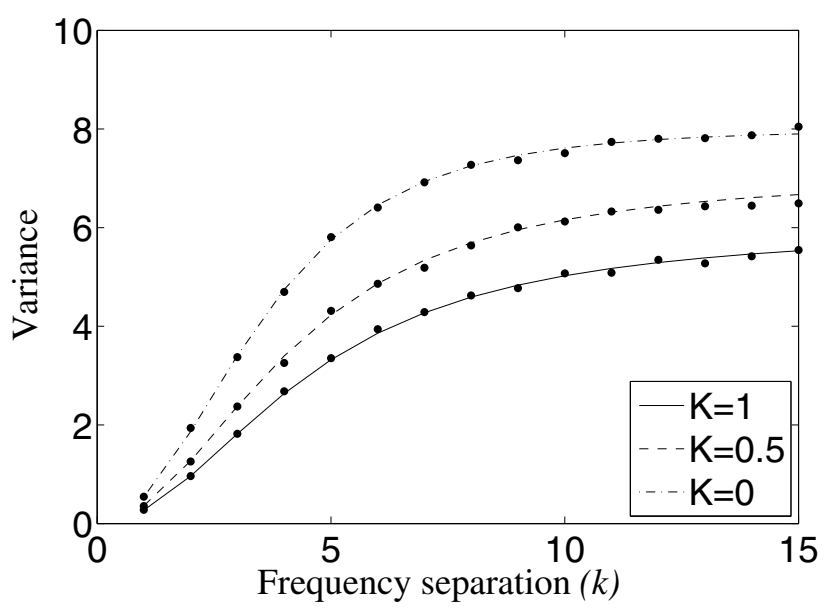

Fig. 6. Variance of $Z_{\text {sum }}$ vs. frequency separation in a $(2,2)$ MIMO OFDM system with exponential PDP, different Rice factors (K) and $\tau_{0} \Delta f=$ 0.01325 .

The motivation for considering this bound is the following. Some simple calculations show that $\left|\rho_{h}(k)\right|^{2}$ is the exact ACF for $Z_{\text {sum }}$ and, since $\lambda_{\max }$ dominates $Z_{\text {sum }}$, it might be expected that the ACF of $Z_{\max }$ is similar. Using this approximate ACF for $\lambda_{\max }$ in (4) gives a simple approximation to $\operatorname{var}\left(Z_{\max }\right)$. This is shown for the exponential case in Fig. 2. Figures 2 and 4 show that this simple approximation gives extremely good results for both the ACF of $\lambda_{\max }$ and $\operatorname{var}\left(Z_{\max }\right)$. Similar results have been obtained over all four power delay profiles and over a range of system sizes. Figure 5 shows this bound applied to $\operatorname{var}\left(Z_{\max }\right)$ and $\operatorname{var}\left(Z_{j}\right)$ in a $(4,4)$ system.

The Rician case is displayed in Fig. 6 for $Z_{\text {sum. }}$. Results are for a $(2,2)$ system with three levels of K-factor. Using (9), the analytical results are shown and verified by simulation. Note that $\operatorname{var}\left(Z_{\text {sum }}\right)=\sigma_{q}^{2} /(1+K)^{2}$, so the result in (9) is scaled. Also, as expected, increasing the line-of-sight strength decreases the variability, and the variance curves are lower as

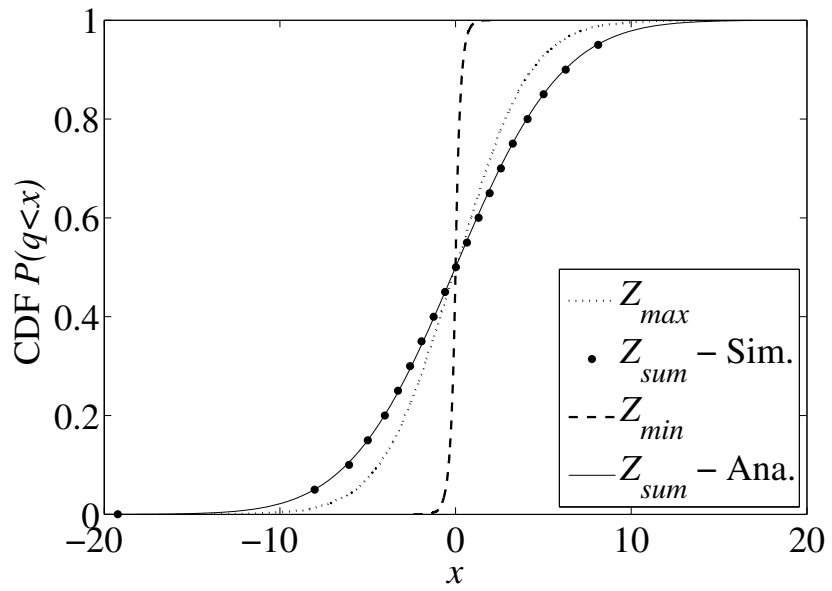

Fig. 7. CDF plots for $Z_{\min }, Z_{\max }$ and $Z_{\text {sum }}$ in a $(4,4)$ MIMO OFDM system with exponential PDP, $\tau_{0} \Delta f=0.01325$ and a separation of 7 bins.

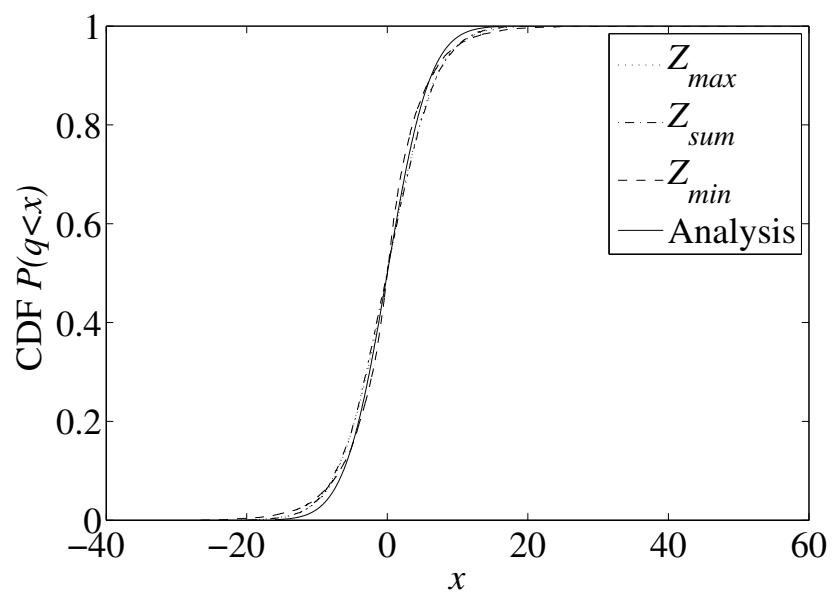

Fig. 8. Scaled CDF plots for $Z_{\min }, Z_{\max }$ and $Z_{\text {sum }}$ in a $(4,4)$ MIMO OFDM system with exponential PDP, $\tau_{0} \Delta f=0.01325$ and a separation of 7 bins.

$K$ increases.

Figures 7 and 8 demonstrate that simple approximations are also possible for the CDFs of $Z_{\max }, Z_{\min }$ and $Z_{\text {sum. }}$. In (19) a CDF was given for $Z_{\max }$, but this involves double numerical integration. The CDF of $Z_{\min }$ can be computed similarly, but the general case, $Z_{j}$, is more awkward. Hence, approximate results are of interest. Figure 7 shows the CDFs of $Z_{\max }, Z_{\min }$ and $Z_{\text {sum }}$ for a $(4,4)$ MIMO system in Rayleigh fading with an exponential power delay profile and a frequency spacing of $k=7$ bins. The CDF for $Z_{\text {sum }}$ is given by (7) and (11) with the remaining CDFs obtained by simulation. As expected, the CDF of $Z_{\min }$ is very sharp and the CDF of $Z_{\text {sum }}$ is the broadest. In Fig. 8 the same scenario is considered, but the variables $Z_{\max }$ and $Z_{\min }$ are scaled so they have the same variance as $Z_{\text {sum. }}$. The new CDFs in Fig. 8 show a reasonably good agreement. Hence, approximate CDFs can be obtained for $Z_{\max }, Z_{\min }$ and therefore for $Z_{j}$ by a simple scaling of the analytical CDFs given for $Z_{\text {sum }}$.

Finally, in Fig. 9, we further verify the simple ACF approx- 


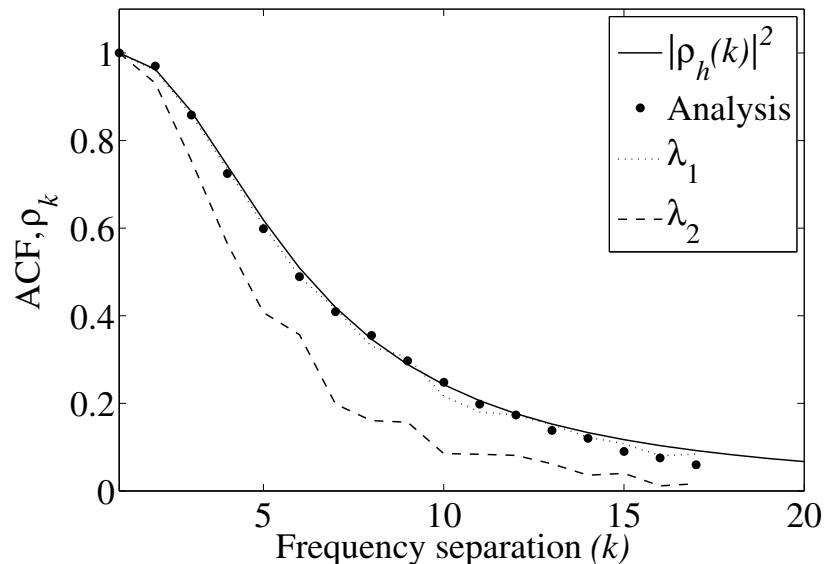

Fig. 9. Frequency autocorrelation function for the eigenvalues in a $(2,2)$ MIMO OFDM system with exponential PDP and $\tau_{0} \Delta f=0.01325$.

imation and show analytical ACF results obtained from (18). A $(2,2)$ system in Rayleigh fading is considered with an exponential power delay profile. We observe that $\left|\rho_{h}(k)\right|^{2}$ provides a close bound, and the analysis is verified by simulation.

\section{CONCLUDING REMARKS}

In MIMO OFDM systems, eigenvalue variation across frequency affects both performance and system design. Such frequency variation also has counterparts in time and space and, although this paper focuses on OFDM, applications also occur in feedback delay and channel estimation problems. We have shown that eigenvalue and link gain changes in frequency can be analyzed and have presented novel results on the distributions and moments of such changes. We have also given expressions for the ACFs of the maximum eigenvalue and the link gain. Of particular interest is the very simple approximation to the ACF of the maximum eigenvalue. This leads to accurate closed-form approximations to the variance and CDF of the eigenvalue differences.

\section{REFERENCES}

[1] P.-H. Kuo, P. J. Smith, L. M. Garth, and N. O'Connell, "Level crossing analysis for MIMO eigenmodes and associated channel metrics," submitted to IEEE Trans. Inform. Theory, March 2007.

[2] P. Ivanis, D. Drajic, and B. Vucetic, "Level crossing rates in transmit beamforming systems," IEEE Commun. Lett., vol. 11, pp. 246-248, Mar. 2007.

[3] E. K. S. Au, S. Jin, M. R. McKay, W. H. Mow, X. Gao, and I. B. Collings, "BER analysis of MIMO-SVD systems with channel estimation error and feedback delay," in Proc. IEEE Int'l. Conf. on Commun., Glasgow, Scotland, 24-28 June 2007, pp. 4375-4380.

[4] H. T. Nguyen, J. B. Andersen, and G. F. Pedersen, "Capacity and performance of MIMO systems under the impact of feedback delay," in Proc. IEEE Int'l. Symp. on Personal, Indoor and Mobile Radio Commun., Aalborg, Denmark, 5-8 Sept 2004, pp. 53-57.

[5] T. J. Willink, "Improving power allocation to MIMO eigenbeams under imperfect channel estimation," IEEE Commun. Lett., vol. 9, pp. 622624, July 2005.

[6] L. J. Cimini Jr., B. Daneshrad, and N. R. Sollenberger, "Capacity and performance of MIMO systems under the impact of feedback delay," in Proc. IEEE Global Telecommun. Conf., London, England, 18-22 Nov 1996, pp. 703-707.
[7] J. Choi and R. W. Heath Jr., "Interpolation based transmit beamforming for MIMO-OFDM with limited feedback," IEEE Trans. Commun., vol. 53, pp. 4125-4135, Nov. 2005.

[8] F. R. Farrokhi, G. J. Lozano, and A. Valenzuela, "Link-optimal spacetime processing with multiple transmit and receive antennas," IEEE Commun. Lett., vol. 5, pp. 85-87, Mar. 2001.

[9] W. C. Jakes, Ed., Microwave Mobile Communications. New York, NY, USA: IEEE Press., 1994.

[10] B. Glance and L. Greenstein, "Frequency-selective fading effects in digital mobile radio with diversity combining," IEEE Trans. Commun., vol. 31, pp. 1085-1094, Sept. 1983.

[11] P. J. Smith and L. M. Garth, "Distribution and characteristic functions for correlated complex Wishart matrices," J. Multivariate Anal., vol. 98, no. 4, pp. 661-677, Apr. 2007.

[12] M. Kang and M. S. Alouini, "Largest eigenvalue of complex Wishart matrices and performance analysis of MIMO MRC systems," IEEE J. Select. Areas Commun., vol. 21, pp. 418-426, Apr. 2003.

[13] A. Zanella, M. Chiani, and M. Z. Win, "On the marginal eigenvalues distribution of Wishart matrices," University of Bologna, Bologna, Italy, IEIIT B0-07-06 Tech. Rep., Dec. 2006.

[14] P. A. Dighe, R. K. Mallik, and S. S. Jamuar, "Analysis of transmitreceive diversity in Rayleigh fading," IEEE Trans. Commun., vol. 51, pp. 694-703, Apr. 2003.

[15] K. H. Biyari and W. C. Lindsey, "Statistical distributions of Hermitian quadratic forms in complex Gaussian variables," IEEE Trans. Inform. Theory, vol. 39, pp. 1076-1082, May 1993.

[16] M. Abramowitz and I. A. Stegun, Eds., Handbook of Mathematical Functions. New York, NY, USA: Dover Publications, 1965.

[17] K. P. Kongara, P.-H. Kuo, P. J. Smith, L. M. Garth, and A. Clark, "Blockbased performance measures for MIMO OFDM beamforming systems,' submitted to IEEE Int'l. Conf. on Commun., 2008.

[18] "Digital C4FM/CQPSK transcever measurement methods," Telecommunications Industry Association (TIA) Standard: TIA-102.CAAA, 1999. 